\title{
Dense hyperthermic intraperitoneal chemotherapy with cisplatin in patients with stage III serous epithelial ovarian cancer: a retrospective study
}

\section{Xiaoli He}

Henan Provincial People's Hospital

\section{Li Wei}

Henan Provincial People's Hospital

Rui Li

Henan Provincial People's Hospital

Shuang Jing

Henan Provincial People's Hospital

\section{Linlin Jia}

Henan Provincial People's Hospital

Danwei Ji

Henan Provincial People's Hosipital

Yali Li

Henan Provincial People's Hospital

\section{Yue Wang}

Henan Provincial People's Hospital

Yongxia Zhu ( $\nabla$ zhuyongxia0409@163.com )

Henan Provincial People's Hospital https://orcid.org/0000-0002-5977-9579

\section{Research Article}

Keywords: Dense hyperthermic intraperitoneal chemotherapy, serous epithelial ovarian cancer, survival, interval debulking surgery

Posted Date: February 15th, 2021

DOI: https://doi.org/10.21203/rs.3.rs-179783/v1

License: (c) (1) This work is licensed under a Creative Commons Attribution 4.0 International License.

Read Full License 


\section{Abstract}

Purpose: To investigate the efficacy and safety of interval debulking surgery (IDS) combined with dense hyperthermic intraperitoneal chemotherapy (HIPEC) with cisplatin in Chinese patients with FIGO stage III serous epithelial ovarian cancer (EOC).

Methods: This retrospective single-center study reviewed the demographic and clinical data of 197 patients with primary FIGO stage III serous EOC who were treated with IDS with $(n=121)$ or without $(n=76$, control group) dense HIPEC between January 2012 and April 2017. The co-primary endpoints were progression-free survival (PFS) and overall survival (OS), and the secondary endpoint was the occurrence of adverse events.

Results: The median PFS was 24 months in the IDS plus dense HIPEC group, whereas it was 19 months in the IDS alone group (hazard ratio [HR] 0.46, 95\% confidence interval [CI]: 0.33-0.65, $p=0.000$ ). The median OS in patients treated with IDS plus dense HIPEC (51 months) was significantly longer than that in patients treated with IDS alone (40 months, HR 0.52, 95\% Cl: 0.35-0.78, $p=0.001$ ). The demographic and preoperative clinical characteristics of these two groups were comparable $(p>0.05)$. In the IDS alone group, no adverse events were recorded in 42 (55.3\%) of the 76 patients, and 14 (18.4\%) patients were reported to have grade III/IV adverse events. In the IDS plus dense HIPEC group, no adverse events were recorded in 55 (45.5\%) of the 121 patients, and 23 (19.0\%) patients were reported to have grade III/IV adverse events. No postoperative deaths occurred within 30 days in either group and neither did severe fatal complications in the IDS plus dense HIPEC group.

Conclusion: IDS plus dense HIPEC with cisplatin in Chinese patients with FIGO stage III serous EOC is associated with improved survival and is reasonably well tolerated by patients.

\section{Introduction}

Epithelial ovarian cancer (EOC) has the highest mortality rate among all gynecological tumors worldwide, causing nearly 22,500 deaths in China in 2015 and 14,335 deaths in the United States in 2018 (Chen et al., 2016; Torre et al., 2018). The most effective treatment for advanced EOC remains primary cytoreductive surgery (CRS) followed by intravenous chemotherapy with carboplatin and paclitaxel (Bristow et al., 2010; Marth et al., 2009). In patients where complete primary cytoreduction is impossible, interval debulking surgery (IDS) can be performed after 2-4 cycles of neoadjuvant chemotherapy (NACT) (Vergote et al., 2010; Vergote et al., 2016; Wright et al., 2016). Peritoneum is the primary site of metastasis of tumor cells from the ovary in advanced EOC patients (Ceresoli et al., 2018; Friedrich et al., 2020). Therefore, intraperitoneal chemotherapy is used to improve outcomes and minimize tumor dissemination and implantation (Ansaloni et al., 2015; Coccolini et al., 2017).

Hyperthermic intraperitoneal chemotherapy (HIPEC) is delivered under hyperthermic conditions and used to increase chemotherapy penetration into peritoneal tumors, including advanced EOC (Riggs et al., 2020). Randomized trials, meta-analyses, and retrospective studies have all shown that HIPEC is feasible 
and associated with prolonged ovarian cancer survival rates (Mendivil et al., 2017; van Driel et al., 2018; Zhang et al., 2019). Some studies have demonstrated that despite the promise of HIPEC, HIPEC did not improve patient survival data compared with therapy without HIPEC (Bristow et al., 2010; Pomel et al., 2010; van Driel et al., 2018). Interestingly, in a meta-analysis of patients with primary EOC, Huo et al. showed that HIPEC combined with CRS was associated with a significantly improved 2-, 3-, 4-, 5-, and 8year OS compared with CRS alone (Huo et al., 2015).

In those studies, different drug and dose ranges of HIPEC were used, which resulted in dissimilar survival times. In some studies, HIPEC was administered only after optimal cytoreductive surgery using cisplatin or a combination of doxorubicin, paclitaxel, or mitomycin C (Cascales Campos et al., 2011; Ceresoli et al., 2018; Delotte et al., 2015; Safra et al., 2014). In other studies, patients received HIPEC with different doses of cisplatin (Antonio et al., 2019; Ba et al., 2014; Deraco et al., 2011; van Driel et al., 2018). Numerous investigations conducted to assess dose dense chemotherapy have shown clinical benefits. For instance, $\mathrm{Ba} \mathrm{M}$, et al. showed that HIPEC procedures with three sessions were performed on the first, third, and fifth days after CRS was comparable to other HIPEC procedures (Ba et al., 2014). Therefore, we referenced the procedure that have three sessions and defined them as Dense HIPEC with cisplatin.

In this single-center study, we sought to retrospectively analyze the role of IDS combined with dense HIPEC with cisplatin in patients with primary FIGO stage III serous EOC after NACT compared with IDS alone.

\section{Materials And Methods}

\section{Patients}

This retrospective study was approved by the Human Ethics Committee of Henan Provincial People's Hospital in China, and written informed consent was obtained from each patient prior to all treatment procedures. All patients in this study were diagnosed with FIGO stage III high-grade serous ovarian cancer, which took surgical findings and histopathological results into consideration, between January 1, 2012 and April 31, 2017 at the Department of Gynecology, Henan Provincial People's Hospital. Staging was done based on the 1988 International FIGO staging system between January 1, 2012 and December 31, 2012, the 2013 International FIGO staging system between January 1, 2013 and December 31, 2013, and the 2014 International FIGO staging system between January 1, 2014 and April 31, 2017 (Mutch and Prat, 2014). The medical records of all patients were reviewed, and all patients were followed up until March $31,2020$.

All patients who demonstrated an Eastern Cooperative Group (ECOG) score 0-1 (Oken et al., 1982; Wenzel et al., 2007) in our study underwent 2-4 cycles of NACT with a combination of carboplatin (area under the curve of 5-6 mg per milliliter per minute, AUC 5-6) and paclitaxel (175 mg per square meter of body-surface area, $175 \mathrm{mg} / \mathrm{m}^{2}$ ). After NACT, IDS was performed using peritonectomy. Complete cytoreductive surgery was defined as surgery that resulted in no visible disease (residual disease 
classification, R0), optimal cytoreductive surgery as surgery that resulted in the presence of one or more residual tumors measuring $\leq 1 \mathrm{~cm}$ in diameter (R1), and incomplete cytoreductive surgery as surgery that resulted in the presence of one or more residual lesions measuring $>1 \mathrm{~cm}$ in diameter (R2) (Chi et al., 2006). The indication for treatment and the HIPEC with cisplatin given were left to the discretion of the treating physician. In this study, regardless of whether satisfactory tumor cell reduction is achieved after IDS (single or more residual lesions $\leq 1 \mathrm{~cm}$ ), HIPEC will be performed with the consent of patients and family members. According to the patient's request and a multidisciplinary discussion among general surgeons, gynecologists, and physicians, patients were divided into two groups: an IDS plus dense HIPEC group and an IDS alone control group.

\section{Inclusion and exclusion criteria}

The inclusion criteria were as follows. (1) Patients aged $\geq 18$ years. (2) Patients that underwent pretreatment and preoperative evaluation with computed tomography (CT) or magnetic resonance imaging (MRI), pelvic ultrasound, and had tumor markers (including cancer antigen 125 [CA125] and human epididymis protein 4 [HE4]) as well as routine blood tests. (3) A pathologic diagnosis confirmed by ascites cytology or biopsy pathology. (4) Patients diagnosed with FIGO stage III serous EOC (highgrade serous ovarian cancer). (5) Patients with normal blood counts, and adequate renal function. (6) Patients with an ECOG performance status of $0-1$.

The exclusion criteria were as follows. (1) Recurrent peritoneal ovarian cancer patients. (2) Patients with previous primary cytoreductive surgery. (3) Patients with the presence of extra-abdominal metastasis. (4) Patients that treated with bevacizumab or poly ADP-ribose polymerase inhibitors. (5) Patients that with poor general health, renal insufficiency, heart failure, or the existence of a lesion in the central nervous system.

\section{IDS plus dense HIPEC group}

After the IDS procedure, dense HIPEC was administered using a custom-developed high-precision body cavity hyperthermic perfusion treatment system (BR-TRG-II, Bright Medical Technology Co., Ltd., Guangzhou, China) through closed procedures (Ba et al., 2014). All patients underwent three sessions of HIPEC, and each session was performed in the general ward on the first, third, and fifth days after surgery with $60 \mathrm{~min}$ of cisplatin $\left(60 \mathrm{mg} / \mathrm{m}^{2}\right)$.

In brief, after cytoreductive surgery, four perfusion chemotherapy catheters with multiple side holes were placed intra-abdominally; one was placed beneath each hemidiaphragm and two were placed in the pelvis. The abdomen was filled with saline that circulated continuously using the HIPEC device. By circulation of the heated saline, an intra-abdominal temperature of $42-43^{\circ} \mathrm{C}$ was maintained. Cisplatin $\left(60 \mathrm{mg} / \mathrm{m}^{2}\right)$ was perfused in equal amounts during each 60 min HIPEC session with a $450-600 \mathrm{~mL} / \mathrm{min}$ flow rate. The total perfusion volume was $3,500-4,500 \mathrm{~mL}$, which was adjusted such that the entire abdomen was exposed to the perfusate. Four abdominal drains were closed during the intraperitoneal intraoperative chemotherapy and opened after $24 \mathrm{~h}$, and all the perfusion liquid was drained prior to 
starting the next session. Four catheters were removed two days after the final HIPEC session. During HIPEC performed in the general ward, patients were attended by a dedicated therapist and monitored with electrocardiograph (ECG) monitoring. Meanwhile, the intervention treatment comprising dezocine and diazepam was administered to the patients. All patients received additional 3-4 cycles of systemic chemotherapy (paclitaxel $175 \mathrm{mg} / \mathrm{m}^{2}$ plus carboplatin AUC 5-6) two weeks after the final HIPEC treatment. Before every HIPEC session, bone marrow, renal and hepatic function, parameters of blood electrolytes, and blood coagulation were examined.

\section{IDS alone control group}

We retrospectively selected FIGO III serous ovarian cancer patients who underwent 2-4 cycles of NACT (paclitaxel $175 \mathrm{mg} / \mathrm{m}^{2}$ plus carboplatin AUC 5-6) followed by IDS. After surgery, 3-4 cycles of intravenous paclitaxel $\left(175 \mathrm{mg} / \mathrm{m}^{2}\right)$ and carboplatin (AUC 5-6) were administered every 21 days.

\section{Study variables}

The following information was reviewed and collected. Patient demographics, medical history, tumor markers, pathologic characteristics, chemotherapy regime including the dose, surgical procedures, tumor debulking status according to the visible residual disease classification (Stuart et al., 2011), the pre- and post-HIPEC lab values (absolute neutrophil count, platelets, hematocrit, and hemoglobin), and HIPEC techniques and drugs, patient adverse events, length of hospital stay, and the cost of hospital data.

\section{Follow-up}

During follow-up after the completion of chemotherapy, physical examinations and measurements of CA125 and HE4 levels were performed every three months for two years and then every six months until the five-year point. CT or MRI was performed at least 1, 6, 12, and 24 months after the last cycle of chemotherapy.

The co-primary endpoints of this study were the survival curves for progression-free survival (PFS) and OS. Clinical, radiologic, and CA125 progression after the initial induction chemotherapy and time to normalization were identified as variables when assessing PFS. OS was defined as the time from the date of initiation of NACT to the date of death from any cause or final follow-up. Safety assessments were performed according to the US National Cancer Institute's Patient-Reported Outcomes version of the Common Terminology Criteria for adverse events (PRO-CTCAE) (Watanabe et al., 2009).

\section{Statistical analyses}

All statistical analyses were performed using SPSS statistical software (version 24.0 for Windows, IBM Corp., Armonk, NY, USA). The initial data analysis was conducted with a descriptive statistical approach. We used the Mann-Whitney $U$ test for continuous variables and the $\chi^{2}$ test for categorical variables. Survival analysis was performed using the Kaplan-Meier approach and the median follow-up was calculated using the reverse Kaplan-Meier approach. Hazard ratios (HRs) were estimated using a Cox 
proportional hazard model with a $95 \%$ Wald confidence interval $(95 \% \mathrm{Cl})$. All $p$ values were two-sided with the level of significance set at $p<0.05$.

\section{Results}

\section{Patient demographics and clinical characteristics}

A total of $197 \mathrm{FIGO}$ stage III serous ovarian cancer patients (high-grade serous ovarian cancer) were reviewed, of whom 121 underwent IDS plus dense HIPEC (IDS plus dense HIPEC group) and 76 underwent IDS (IDS alone control group). There were no differences between the two groups in terms of patient age $(p=0.337)$ or body mass index (BMl; $p=0.277)$. Optimal cytoreductive surgery ( $\mathrm{R} 0+\mathrm{R} 1)$ was achieved in $113(93.4 \%)$ patients in the IDS plus dense HIPEC group and 70 (92.1\%) patients in the IDS alone control group $(p=0.734)$. Although there were no differences in disease grade $(p=0.337)$, the median duration of hospitalization after surgery was significantly different between the two groups $(p=0.001)$. In the IDS plus dense HIPEC group, patients were treated with a mean of $2.95(\mathrm{SD}=0.62)$ cycles of NACT compared with $2.86(S D=0.60)$ cycles in the IDS alone group $(p=0.442)$. The individual surgery groups' demographic and clinical characteristics are described in Table 1. 
Table 1

Demographic and clinical characteristics of patient between groups

\begin{tabular}{|c|c|c|c|}
\hline Variable & $\begin{array}{l}\text { IDS + HIPEC }(n \\
=121)\end{array}$ & $\begin{array}{l}\text { IDS alone }(n \\
=76)\end{array}$ & value \\
\hline \multicolumn{4}{|l|}{ Demographic mean (SD) } \\
\hline Age & $58.38(10.89)$ & $\begin{array}{l}55.20 \\
(10.87)\end{array}$ & $0.337^{a}$ \\
\hline $\mathrm{BMI}\left(\mathrm{kg} / \mathrm{m}^{2}\right)(\mathrm{SD})$ & $26.77(2.78)$ & $26.65(2.40)$ & $0.277^{a}$ \\
\hline Neo-adjuvant chemotherapy cycles, n (\%) & & & $0.442^{b}$ \\
\hline Two cycles & $28(23.1)$ & $20(26.3)$ & \\
\hline Three cycles & $74(61.2)$ & $47(61.8)$ & \\
\hline Four cycles & $19(15.7)$ & $9(11.8)$ & \\
\hline Cyto-reductive surgery, n (\%) & & & $0.734^{b}$ \\
\hline Optimal (R0 + R1) & $113(93.4)$ & $70(92.1)$ & \\
\hline Sub-optimal (residual disease $>1 \mathrm{~cm}$ ) & $8(6.6)$ & $6(7.9)$ & \\
\hline Bowel resection, n (\%) & & & $0.523^{b}$ \\
\hline No bowel resection performed & $101(83.5)$ & $66(86.8)$ & \\
\hline Bowel resection performed & $20(16.5)$ & $10(13.2)$ & \\
\hline $\begin{array}{l}\text { Mean duration of hospitalization-days (SD) after } \\
\text { surgery }\end{array}$ & $8.91(1.89)$ & $8.07(1.80)$ & $0.001^{\mathrm{a}}$ \\
\hline $\begin{array}{l}\text { Median time to postoperative systemic treatment with } \\
\text { chemotherapy (SD) }\end{array}$ & $22(2.37)$ & $15(1.87)$ & $0.001^{\mathrm{a}}$ \\
\hline Performance status (ECOG), n (\%) & & & $0.980^{\mathrm{b}}$ \\
\hline 0 & $38(31.4)$ & $24(31.6)$ & \\
\hline 1 & $83(68.6)$ & $52(68.4)$ & \\
\hline TC chemotherapy cycles after IDS (\%) & & & $0.015^{b}$ \\
\hline 3 cycles & $6(5.0)$ & $5(6.6)$ & \\
\hline 4 cycles & $58(47.9)$ & $49(64.5)$ & \\
\hline 5 cycles & $57(47.1)$ & $22(28.9)$ & \\
\hline
\end{tabular}

aAnalysed using an Independent-Samples t-test; ${ }^{b}$ Analysed using the Mann-Whitney U test. ECOG:

Eastern Collaborative Oncology Group 


\begin{tabular}{|llll|}
\hline Variable & $\begin{array}{l}\text { IDS + HIPEC ( } \\
=121)\end{array}$ & $\begin{array}{l}\text { IDS alone }(\mathbf{n} \\
\mathbf{=} 76)\end{array}$ & $\begin{array}{l}\text { p- } \\
\text { value }\end{array}$ \\
\hline Total of TC cycles (SD) & $6.73(0.53)$ & $6.48(0.66)$ & $0.047^{\mathrm{b}}$ \\
\hline $\begin{array}{l}\text { aAnalysed using an Independent-Samples t-test; }{ }^{\mathrm{b}} \text { Analysed using the Mann-Whitney U test. ECOG: } \\
\text { Eastern Collaborative Oncology Group }\end{array}$ & & \\
\hline
\end{tabular}

\section{PFS and OS}

The median follow-up was 46 and 38 months in the IDS plus dense HIPEC and IDS alone group, respectively. In the IDS plus dense HIPEC group, the median PFS was 24 (95\% Cl: 21.38-26.62) months compared with 19 (95\% Cl: 17.81-20.19) months in the IDS alone group $(p=0.000)$, suggesting that the IDS plus dense HIPEC group experienced a significantly longer PFS than the IDS alone group (Fig. 1). Of the 197 patients, 142 patients ( $72.1 \%$ of the recurrences) were detected based on imaging and 55 patients (27.9\%) were detected based on an increase in CA125 levels alone (more than $35 \mathrm{U} / \mathrm{mL}$ ). Overall, 69 of the 121 patients $(52.02 \%)$ in the IDS plus dense HIPEC group and 55 of the 76 patients $(72.37 \%)$ in the IDS alone group died during the follow-up period. The median OS was 51 (95\% Cl: 42.73-59.27) months in the IDS plus dense HIPEC group and 40 (95\% Cl: $37.59-42.41)$ months in the IDS group ( $p=$ 0.001 , Fig. 2). Significant differences were observed between the groups in both the median PFS (HR $0.46,95 \% \mathrm{Cl}: 0.33-0.65, p=0.000)$ and median OS (HR 0.52, 95\% Cl: $0.35-0.78, p=0.001$ ).

\section{Adverse events due to HIPEC}

The median duration of hospitalization after surgery was $8.91(S D=1.89)$ days in the IDS plus dense HIPEC group and $8.07(S D=1.80)$ days in the IDS alone group $(p=0.000)$. Patients treated with IDS plus dense HIPEC received a mean of $4.4(S D=0.59)$ cycles of carboplatin and paclitaxel chemotherapy after a median duration of 22 days of postoperative systemic treatment with chemotherapy. Patients treated with IDS alone underwent a mean of $4.2(S D=0.56)$ cycles after a median duration of 15 days of postoperative systemic treatment with chemotherapy $(p=0.000)$. In the IDS plus HIPEC group, a total of seven patients could not complete three sessions of HIPEC, implying they only underwent two sessions of HIPEC. Two patients developed fever and had a body temperature as high as $39^{\circ} \mathrm{C}$ for three days, and four patients had intestinal obstruction symptoms after hyperthermic perfusion on both sides. In one patient, the third session of HIPEC was terminated because of intestinal anastomosis bleeding.

In the IDS plus HIPEC group, no adverse events were recorded in 55 (45.5\%) of the 121 patients in the group, and $23(19.0 \%)$ of the 121 patients were reported to have grade III or IV adverse events. Conversely, in the IDS alone group, no adverse events were recorded for $42(55.3 \%)$ of the 76 patients, and 14 (18.4\%) were reported to have grade III-IV adverse events. The main adverse events observed in this study are summarized in Tables 2 and 3. The most common grade I-II adverse event observed in this study was an electrolyte disturbance, and the most common grade III-IV adverse event was ileus. All HIPEC procedures were performed in the general ward and not in the intensive care unit (ICU). No deaths occurred within the 
first 30 days postoperatively in the two groups of patients and neither did severe fatal complications in the IDS plus dense HIPEC group.

\section{Table 2}

Adverse events for the ovarian cancer patients treated with IDS plus HIPEC $(n=121)$

\begin{tabular}{|lll|}
\hline Adverse events $\mathbf{n}(\%)$ & Grade I or II & Grade III or IV \\
\hline Haematology & $21(17.4)$ & 0 \\
\hline Leucocytopenia & $9(7.4)$ & 0 \\
\hline Thrombocytopenia & $30(24.8)$ & $1(0.8)$ \\
\hline Anemia & $38(31.4)$ & $4(3.3)$ \\
\hline Electrolyte disturbance* & $10(8.3)$ & $3(2.5)$ \\
\hline Thromboembolism & $27(22.3)$ & 0 \\
\hline Nausea & $16(13.2)$ & 0 \\
\hline Emesis & $5(4.1)$ & 0 \\
\hline Nephrotoxicity & $26(21.5)$ & $2(1.7)$ \\
\hline Abdominal pain & $6(5.0)$ & $10(8.3)$ \\
\hline Ileus & $4(3.3)$ & $2(1.7)$ \\
\hline Anastomotic hemorrhage & $15(12.4)$ & $3(2.5)$ \\
\hline Infection & & \\
\hline $\begin{array}{l}\text { Electrolyte disturbances included hyponatremia, hypochloridemia, hypokalemia, hypocalcemia, } \\
\text { hypomagnesemia and hypophosphatemia. Infection included Lung infection, Urinary tract infection, } \\
\text { Urethral infection, Stoma site infection, Sepsis, Bacteremia, Abdominal infection. }\end{array}$ \\
\hline
\end{tabular}


Table 3

Adverse events for the ovarian cancer patients treated with IDS alone $(n=76)$

\begin{tabular}{|lll|}
\hline Adverse Events $\mathbf{n}(\%)$ & Grade I or II & Grade III or IV \\
\hline Haematology & & 0 \\
\hline Leucocytopenia & $2(2.6)$ & 0 \\
\hline Thrombocytopenia & 0 & $2(2.6)$ \\
\hline Anemia & $18(23.7)$ & $2(2.6)$ \\
\hline Electrolyte disturbance* & $20(26.3)$ & $2(2.6)$ \\
\hline Thromboembolism & $5(6.6)$ & 0 \\
\hline Nausea & $15(19.7)$ & 0 \\
\hline Emesis & $9(11.8)$ & 0 \\
\hline Nephrotoxicity & 0 & 0 \\
\hline Abdominal pain & $13(17.1)$ & $5(6.6)$ \\
\hline lleus & $2(2.6)$ & 0 \\
\hline anastomotic hemorrhage & $3(3.9)$ & $3(3.9)$ \\
\hline Infection & $11(14.5)$ & \\
\hline $\begin{array}{l}\text { Electrolyte disturbances included hyponatremia, hypochloridemia, hypokalemia, hypocalcemia, } \\
\text { hypomagnesemia and hypophosphatemia. Infection included Lung infection, Urinary tract infection, }\end{array}$ \\
\hline Urethral infection, Stoma site infection, Sepsis, Bacteremia, Abdominal infection. \\
\hline
\end{tabular}

\section{Discussion}

In most cases of advanced ovarian cancer, peritoneal metastasis is the primary site of spread and cause of treatment failure (Friedrich et al., 2020). Several randomized clinical trials have shown that postoperative intraperitoneal and intravenous chemotherapy improved survival in patients with optimally resected stage IIIC ovarian cancer compared with intravenous chemotherapy alone (Armstrong et al., 2006; Markman et al., 2001; Tewari et al., 2015; Wenzel et al., 2007). Although the survival benefits of intraperitoneal chemotherapy have been reported in GOG172 (Armstrong et al., 2006), the toxicity and significantly high incidence of side effects have prevented this treatment from being widely adopted in clinical practice (Riggs et al., 2020; Wenzel et al., 2007). In recent years, the application of HIPEC to ovarian cancer has garnered interest because of its advantages over standard intraperitoneal chemotherapy (Riggs et al., 2020).

Nevertheless, the prognostic value of HIPEC in ovarian cancer has been controversial. Some studies have demonstrated that HIPEC does not improve OS compared with therapy without HIPEC (Mendivil et al., 2017). However, an increasing number of studies have demonstrated that the addition of HIPEC 
significantly improves the prognosis of ovarian cancer (Ba et al., 2014; Fagotti et al., 2012; Kim et al., 2010; Rettenmaier et al., 2015; Torre et al., 2018), especially in randomized controlled trials (Pomel et al., 2010; Spiliotis et al., 2015; van Driel et al., 2018). The drug and dose range of HIPEC are important considerations that can affect survival times. In some studies, HIPEC was performed only after optimal cytoreductive surgery, either as an open procedure or a closed system, which used cisplatin or a combination of doxorubicin, paclitaxel, or mitomycin C (Cascales Campos et al., 2011; Ceresoli et al., 2018; Delotte et al., 2015; Safra et al., 2014). In other studies, patients underwent HIPEC with different doses of cisplatin (Ba et al., 2014; Deraco et al., 2011; van Driel et al., 2018). In the study by Antonio et al., patients were treated with HIPEC and paclitaxel alone (Antonio et al., 2019). Hence, several investigations have shown that dose dense chemotherapy provides clinical benefits. Therefore, this study focused on the feasibility of dense HIPEC, which involves increasing the rate of chemotherapy with three sessions of HIPEC, in FIGO stage III serous EOC.

In the present study, the median PFS was 24 months in the IDS plus dense HIPEC group, which was significantly better than the 19 months observed in the IDS alone control group, suggesting the feasibility of dense HIPEC in patients with FIGO stage III serous EOC. Two groups of patients in this study received similar preoperative treatment and had the same disease stage and pathological type. No significant differences in cytoreductive surgery, pathological grade, or the total number of cycles of systematic intravenous chemotherapy existed between the two groups. Mendivil et al. reported improved PFS with HIPEC compared with intravenous chemotherapy (25.1 months versus 20.0 months, respectively; $p=$ 0.024) (Mendivil et al., 2017), which corroborates the results of this study. Similarly, a randomized, controlled, open-label trial also documented an increased PFS (14.2 months versus 10.7 months) in cohorts treated with HIPEC chemotherapy (van Driel et al., 2018).

Although previous studies reported favorable PFS results, OS benefits have conventionally been considered as the most dependable endpoint in assessing cancer-related treatments. In a multicenter, open-label, phase 3 trial (van Driel et al., 2018) 245 patients who received NACT were randomly assigned to undergo interval cytoreductive surgery either with or without HIPEC with cisplatin $\left(100 \mathrm{mg} / \mathrm{m}^{2}\right)$. A significant improvement in OS was seen: 45.7 months versus 33.9 months in the surgery plus HIPEC and surgery alone group, respectively. Notably, our results showing a median OS of 51.0 months for the IDS plus dense HIPEC group versus 40.0 months for the IDS alone group. Nevertheless, a recent retrospective case-control study of 56 patients treated for primary advanced ovarian cancer who underwent interval surgery with or without HIPEC demonstrated that although OS was better in the case group than in the control group ( $p=0.048$ ), PFS was not significantly different ( 13.2 months versus 13.9 months, $p=0.454$ ) (Ceresoli et al., 2018). Conversely, another retrospective case-control study showed that PFS was significantly longer with HIPEC than without, but no OS advantages were identified. The differences in these results may have several explanations. First, follow-up differed in the two studies, and a shorter duration was inherent to the HIPEC group (Mendivil et al., 2017). Second, the studies were retrospectively evaluated, and selection bias may have influenced the outcomes (Mendivil et al., 2017). Third, a relatively 
small number of patients were included to obtain the results. Last, the rate of the peritoneal metastasis differed, which is a critical determinant of survival in advanced ovarian cancer (Ceresoli et al., 2018).

Unsurprisingly, we observed a reasonable toxicity profile in the patients receiving dense HIPEC. No grade IV adverse events were recorded in any of the 121 patients, and no adverse events were recorded in 55 (45.5\%) patients, however, 43 patients developed grade II nausea during the three sessions of HIPEC. Notably, we did not observe any grade III-IV leukocytopenia, thrombocytopenia, or renal failure. Similarly, several retrospective studies did not report any evidence of grade III-IV toxicity in their experience of treating advanced ovarian cancer patients with HIPEC (Dharmadhikari et al., 2014; Mendivil et al., 2017).

To the best of our knowledge, HIPEC as a single treatment has only been administered at the end of cytoreductive surgery in the operating room or the ICU (Ba et al., 2014; Ceresoli et al., 2018; Mendivil et al., 2017; van Driel et al., 2018), thus increasing not only patient safety and comfort but also medical costs (Lim et al., 2019). However, in our center, dense HIPEC is delivered with cisplatin after cytoreductive surgery in the general ward, and no adverse events were found to be associated with this. Furthermore, the patient tolerability was excellent. The median time of postoperative systemic treatment with chemotherapy was 22 days, which was significantly lower than the 33-49 days reported in previous studies (Ceresoli et al., 2018; van Driel et al., 2018).

In addition, specific pathologic subtypes affect the prognosis of advanced ovarian cancer. To reduce the impact of pathological type and disease stage on survival in the present study, we only included FIGO stage III high-grade serous EOC patients. Several limitations should be considered in this study. First, the retrospective nature of our analysis reduced our ability to draw reliable conclusions. Second, although NACT is increasingly used as the primary treatment for advanced ovarian cancer (Mueller et al., 2016), patients who undergo primary debulking surgery have a significant survival benefit compared with those who undergo NACT. Third, this study showed that significant benefits in OS and PFS were associated with dense HIPEC, however, multivariate analyses were not performed to elucidate the risk factors of prognosis nor evaluate the impact of optimal cytoreductive surgery, CA125 level, age, and other variables. In a retrospective multicenter study of 78 patients by Le Saux, et al., the univariate analysis demonstrated that age $\geq 50$ years, peritoneal cancer index $(\mathrm{PCl}) \leq 8$, and CA125 levels $<100$ were significantly associated with long-term survival in patients with EOC following cytoreductive surgery and HIPEC (Le Saux et al., 2018). However, our data were limited to optimal or suboptimal cytoreductive surgery (Chi et al., 2006), and it may have been preferable to further stratify the surgery categories into complete (R0), optimal < $1 \mathrm{~cm}$ (R1), and suboptimal (R2) groups (Armstrong et al., 2006).

At present, there are many ongoing randomized Phase 2 and 3 trials involving primary and recurrent disease as well as patients receiving NACT (Cowan et al., 2017). Many of these trials intend to assess the impact of adding HIPEC to cytoreductive surgery on PFS and OS (Cowan et al., 2017) as well as on morbidity, quality of life, and pharmacokinetics (Helm, 2012). However, to the best of our knowledge, few randomized controlled clinical trials of dense HIPEC training and administration with the agents and protocols used in our study are ongoing. 


\section{Conclusions}

This retrospective study of FIGO stage III serous EOC patients indicates that IDS plus dense HIPEC results in longer survival than IDS alone, and this treatment is reasonably well tolerated by patients. Future trials investigating the efficacy and safety of dense HIPEC plus debulking surgery in a similar setting are required.

\section{Declarations}

\section{Ethics approval and consent to participate}

This retrospective study was approved by the Human Ethics Committee of Henan Provincial People's Hospital in China, and written informed consent was obtained from each patient prior to all treatment procedures. Patients were informed that the resected specimens were stored by the hospital and potentially used for scientific research, and that privacy would be maintained.

\section{Consent for publication}

Not applicable.

\section{Availability of data and materials}

The datasets used or analyzed during the current study are available from the corresponding author on reasonable request.

\section{Competing interests}

The authors declare that they have no conflict of interest.

\section{Funding}

This work was supported by the Health Commission of Henan Province: Hyperthermic intraperitoneal chemotherapy in borderline mucous tumor under Grant [number 2018020423]. None of the funding agencies had a role in designing the study, writing the manuscript, nor the collection, analysis, and interpretation of data.

\section{Authors' contributions}

All authors contributed to the study conception and design. $\mathrm{XH}$ : planning and study design, study execution, and writing-initial draft. $L W$ and $Y Z$ : Statistical analysis/interpretation, and writing-final revision. RL: Planning and study design; $S J, L J, D J$ and $Y L$ : Date collection and study execution. $Y W$ : Writing-final revision. All authors have read and approve the final version.

\section{Acknowledgments}


The authors thank all the patients and their family members for their interest and cooperation in the study.

\section{References}

1. Ansaloni L, Coccolini F, Morosi L, Ballerini A, Ceresoli M, Grosso G, Bertoli P, Busci LM, Lotti M, Cambria F, et al. Pharmacokinetics of concomitant cisplatin and paclitaxel administered by hyperthermic intraperitoneal chemotherapy to patients with peritoneal carcinomatosis from epithelial ovarian cancer. Br J Cancer. 2015;112:306-12.

2. Antonio CCP, Francisco-Cristobal MC, Alida GG, Susana SG, Israel M, Rafael M, David PV, Pedro V, Fernando P, Elena G, et al. (2019). Upfront citorreduction and hyperthermic intraperitoneal chemotherapy with paclitaxel in patients with stage III-C serous epithelial ovarian cancer. Clin Exp Metastasis.

3. Armstrong DK, Bundy B, Wenzel L, Huang HQ, Baergen R, Lele S, Copeland LJ, Walker JL, Burger RA. Intraperitoneal cisplatin and paclitaxel in ovarian cancer. N Engl J Med. 2006;354:34-43.

4. Ba M, Long H, Zhang X, Tang Y, Wu Y, Yu F, Wang S, Cui S. Different sequential approaches of cytoreductive surgery and hyperthermic intraperitoneal chemotherapy in treating ovarian cancer with malignant ascites. J Cancer Res Clin Oncol. 2014;140:1497-506.

5. Bristow RE, Palis BE, Chi DS, Cliby WA. The National Cancer Database report on advanced-stage epithelial ovarian cancer: impact of hospital surgical case volume on overall survival and surgical treatment paradigm. Gynecol Oncol. 2010;118:262-7.

6. Cascales Campos PA, Gil Martinez J, Galindo Fernandez PJ, Gil Gomez E, Martinez Frutos IM, Paricio $P, P$. Perioperative fast track program in intraoperative hyperthermic intraperitoneal chemotherapy (HIPEC) after cytoreductive surgery in advanced ovarian cancer. Eur J Surg Oncol. 2011;37:543-8.

7. Ceresoli M, Verrengia A, Montori G, Busci L, Coccolini F, Ansaloni L, Frigerio L. Effect of cytoreductive surgery and hyperthermic intraperitoneal chemotherapy on relapse pattern in primary epithelial ovarian cancer: a propensity score based case-control study. J Gynecol Oncol. 2018;29:e53.

8. Chen W, Zheng R, Baade PD, Zhang S, Zeng H, Bray F, Jemal A, Yu XQ, He J. Cancer statistics in China, 2015. Cancer J Clin. 2016;66:115-32.

9. Chi DS, Eisenhauer EL, Lang J, Huh J, Haddad L, Abu-Rustum NR, Sonoda Y, Levine DA, Hensley M, Barakat RR. (2006). What is the optimal goal of primary cytoreductive surgery for bulky stage IIIC epithelial ovarian carcinoma (EOC)? Gynecologic oncology 103, 559-564.

10. Coccolini F, Acocella F, Morosi L, Brizzola S, Ghiringhelli M, Ceresoli M, Davoli E, Ansaloni L, D'Incalci M, Zucchetti M. High Penetration of Paclitaxel in Abdominal Wall of Rabbits after Hyperthermic Intraperitoneal Administration of Nab-Paclitaxel Compared to Standard Paclitaxel Formulation. Pharm Res. 2017;34:1180-6.

11. Cowan RA, O'Cearbhaill RE, Zivanovic O, Chi DS. Current status and future prospects of hyperthermic intraoperative intraperitoneal chemotherapy (HIPEC) clinical trials in ovarian cancer. Int $\mathrm{J}$ 
Hyperthermia. 2017;33:548-53.

12. Delotte J, Arias T, Guerin O, Boulahssass R, Bereder I, Bongain A, Benchimol D, Bereder JM. Hyperthermic intraperitoneal chemotherapy for the treatment of recurrent ovarian cancer in elderly women. Acta Obstet Gynecol Scand. 2015;94:435-9.

13. Deraco M, Kusamura S, Virzi S, Puccio F, Macri A, Famulari C, Solazzo M, Bonomi S, lusco DR, Baratti D. Cytoreductive surgery and hyperthermic intraperitoneal chemotherapy as upfront therapy for advanced epithelial ovarian cancer: multi-institutional phase-II trial. Gynecol Oncol. 2011;122:21520.

14. Dharmadhikari N, Shah R, Jagannath P. Initial experience with hyperthermic intra peritoneal chemotherapy and cytoreductivesurgery. Indian J Cancer. 2014;51:189-92.

15. Fagotti A, Costantini B, Petrillo M, Vizzielli G, Fanfani F, Margariti PA, Turco LC, Piovano E, Scambia G. Cytoreductive surgery plus HIPEC in platinum-sensitive recurrent ovarian cancer patients: a casecontrol study on survival in patients with two year follow-up. Gynecol Oncol. 2012;127:502-5.

16. Friedrich M, Zinn W, Kolnsberg L, Kraft C, Kuhn W. Hyperthermic Intraperitoneal Chemotherapy (HIPEC) for Ovarian Cancer: Evaluation of Side Effects in a Single Institution Cohort. Anticancer research. 2020;40:1481-6.

17. Helm CW. Current status and future directions of cytoreductive surgery and hyperthermic intraperitoneal chemotherapy in the treatment of ovarian cancer. Surg Oncol Clin N Am. 2012;21:645-63.

18. Huo YR, Richards A, Liauw W, Morris DL. Hyperthermic intraperitoneal chemotherapy (HIPEC) and cytoreductive surgery (CRS) in ovarian cancer: A systematic review and meta-analysis. Eur J Surg Oncol. 2015;41:1578-89.

19. Kim JH, Lee JM, Ryu KS, Lee YS, Park YG, Hur SY, Lee KH, Lee SH, Kim SJ. Consolidation hyperthermic intraperitoneal chemotherapy using paclitaxel in patients with epithelial ovarian cancer. J Surg Oncol. 2010;101:149-55.

20. Le Saux O, Decullier E, Freyer G, Glehen O, Bakrin N. Long-term survival in patients with epithelial ovarian cancer following cytoreductive surgery and hyperthermic intraperitoneal chemotherapy (HIPEC). Int J Hyperthermia. 2018;35:652-7.

21. Lim SL, Havrilesky LJ, Habib AS, Secord AA. Cost-effectiveness of hyperthermic intraperitoneal chemotherapy (HIPEC) at interval debulking of epithelial ovarian cancer following neoadjuvant chemotherapy. Gynecol Oncol. 2019;153:376-80.

22. Markman M, Bundy BN, Alberts DS, Fowler JM, Clark-Pearson DL, Carson LF, Wadler S, Sickel J. Phase III trial of standard-dose intravenous cisplatin plus paclitaxel versus moderately high-dose carboplatin followed by intravenous paclitaxel and intraperitoneal cisplatin in small-volume stage III ovarian carcinoma: an intergroup study of the Gynecologic Oncology Group, Southwestern Oncology Group, and Eastern Cooperative Oncology Group. Journal of clinical oncology: official journal of the American Society of Clinical Oncology. 2001;19:1001-7. 
23. Marth C, Hiebl S, Oberaigner W, Winter R, Leodolter S, Sevelda P. Influence of department volume on survival for ovarian cancer: results from a prospective quality assurance program of the Austrian Association for Gynecologic Oncology. Int J Gynecol Cancer. 2009;19:94-102.

24. Mendivil AA, Rettenmaier MA, Abaid LN, Brown JV 3rd, Mori KM, Lopez KL, Goldstein BH. Consolidation hyperthermic intraperitoneal chemotherapy for the treatment of advanced stage ovarian carcinoma: a 3 year experience. Cancer Chemother Pharmacol. 2017;80:405-10.

25. Mueller JJ, Zhou QC, lasonos A, O'Cearbhaill RE, Alvi FA, El Haraki A, Eriksson AG, Gardner GJ, Sonoda Y, Levine DA, et al. Neoadjuvant chemotherapy and primary debulking surgery utilization for advanced-stage ovarian cancer at a comprehensive cancer center. Gynecol Oncol. 2016;140:436-42.

26. Mutch DG, Prat J. 2014 FIGO staging for ovarian, fallopian tube and peritoneal cancer. Gynecol Oncol. 2014;133:401-4.

27. Oken MM, Creech RH, Tormey DC, Horton J, Davis TE, McFadden ET, Carbone PP. Toxicity and response criteria of the Eastern Cooperative Oncology Group. Am J Clin Oncol. 1982;5:649-55.

28. Pomel C, Ferron G, Lorimier G, Rey A, Lhomme C, Classe JM, Bereder JM, Quenet F, Meeus P, Marchal $F$, et al. Hyperthermic intra-peritoneal chemotherapy using oxaliplatin as consolidation therapy for advanced epithelial ovarian carcinoma. Results of a phase II prospective multicentre trial. CHIPOVAC study. Eur J Surg Oncol. 2010;36:589-93.

29. Rettenmaier MA, Mendivil AA, Abaid LN, lii B, Wilcox JV, A.M., and Goldstein BH. Consolidation hyperthermic intraperitoneal chemotherapy and maintenance chemotherapy following laparoscopic cytoreductive surgery in the treatment of ovarian carcinoma. Int J Hyperthermia. 2015;31:8-14.

30. Riggs MJ, Pandalai PK, Kim J, Dietrich CS. (2020). Hyperthermic Intraperitoneal Chemotherapy in Ovarian Cancer. Diagnostics (Basel) 10.

31. Safra T, Grisaru D, Inbar M, Abu-Abeid S, Dayan D, Matceyevsky D, Weizman A, Klausner JM. Cytoreduction surgery with hyperthermic intraperitoneal chemotherapy in recurrent ovarian cancer improves progression-free survival, especially in BRCA-positive patients- a case-control study. J Surg Oncol. 2014;110:661-5.

32. Spiliotis J, Halkia E, Lianos E, Kalantzi N, Grivas A, Efstathiou E, Giassas S. Cytoreductive surgery and HIPEC in recurrent epithelial ovarian cancer: a prospective randomized phase III study. Ann Surg Oncol. 2015;22:1570-5.

33. Stuart GC, Kitchener H, Bacon M, duBois A, Friedlander M, Ledermann J, Marth C, Thigpen T, Trimble E. (2011). 2010 Gynecologic Cancer InterGroup (GCIG) consensus statement on clinical trials in ovarian cancer: report from the Fourth Ovarian Cancer Consensus Conference. Int J Gynecol Cancer $21,750-755$.

34. Tewari D, Java JJ, Salani R, Armstrong DK, Markman M, Herzog T, Monk BJ, Chan JK. Long-term survival advantage and prognostic factors associated with intraperitoneal chemotherapy treatment in advanced ovarian cancer: a gynecologic oncology group study. Journal of clinical oncology: official journal of the American Society of Clinical Oncology. 2015;33:1460-6. 
35. Torre LA, Trabert B, DeSantis CE, Miller KD, Samimi G, Runowicz CD, Gaudet MM, Jemal A, Siegel RL. Ovarian cancer statistics, 2018. CA Cancer J Clin. 2018;68:284-96.

36. van Driel WJ, Koole SN, Sikorska K, Schagen van Leeuwen, JH, Schreuder HWR, Hermans RHM, de Hingh I, van der Velden J, Arts HJ, Massuger L, et al. Hyperthermic Intraperitoneal Chemotherapy in Ovarian Cancer. N Engl J Med. 2018;378:230-40.

37. Vergote I, Trope CG, Amant F, Kristensen GB, Ehlen T, Johnson N, Verheijen RH, van der Burg ME, Lacave AJ, Panici PB, et al. Neoadjuvant chemotherapy or primary surgery in stage IIIC or IV ovarian cancer. N Engl J Med. 2010;363:943-53.

38. Vergote IB, Van Nieuwenhuysen E, Vanderstichele A. How to Select Neoadjuvant Chemotherapy or Primary Debulking Surgery in Patients With Stage IIIC or IV Ovarian Carcinoma. Journal of clinical oncology: official journal of the American Society of Clinical Oncology. 2016;34:3827-8.

39. Watanabe H, Okada M, Kaji Y, Satouchi M, Sato Y, Yamabe Y, Onaya H, Endo M, Sone M, Arai Y. [New response evaluation criteria in solid tumours-revised RECIST guideline (version 1.1)]. Gan To Kagaku Ryoho. 2009;36:2495-501.

40. Wenzel LB, Huang HQ, Armstrong DK, Walker JL, Cella D. Health-related quality of life during and after intraperitoneal versus intravenous chemotherapy for optimally debulked ovarian cancer: a Gynecologic Oncology Group Study. Journal of clinical oncology: official journal of the American Society of Clinical Oncology. 2007;25:437-43.

41. Wright AA, Bohlke K, Armstrong DK, Bookman MA, Cliby WA, Coleman RL, Dizon DS, Kash JJ, Meyer LA, Moore KN, et al. Neoadjuvant chemotherapy for newly diagnosed, advanced ovarian cancer: Society of Gynecologic Oncology and American Society of Clinical Oncology Clinical Practice Guideline. Gynecol Oncol. 2016;143:3-15.

42. Zhang G, Zhu Y, Liu C, Chao G, Cui R, Zhang Z. The prognosis impact of hyperthermic intraperitoneal chemotherapy (HIPEC) plus cytoreductive surgery (CRS) in advanced ovarian cancer: the metaanalysis. J Ovarian Res. 2019;12:33.

\section{Figures}




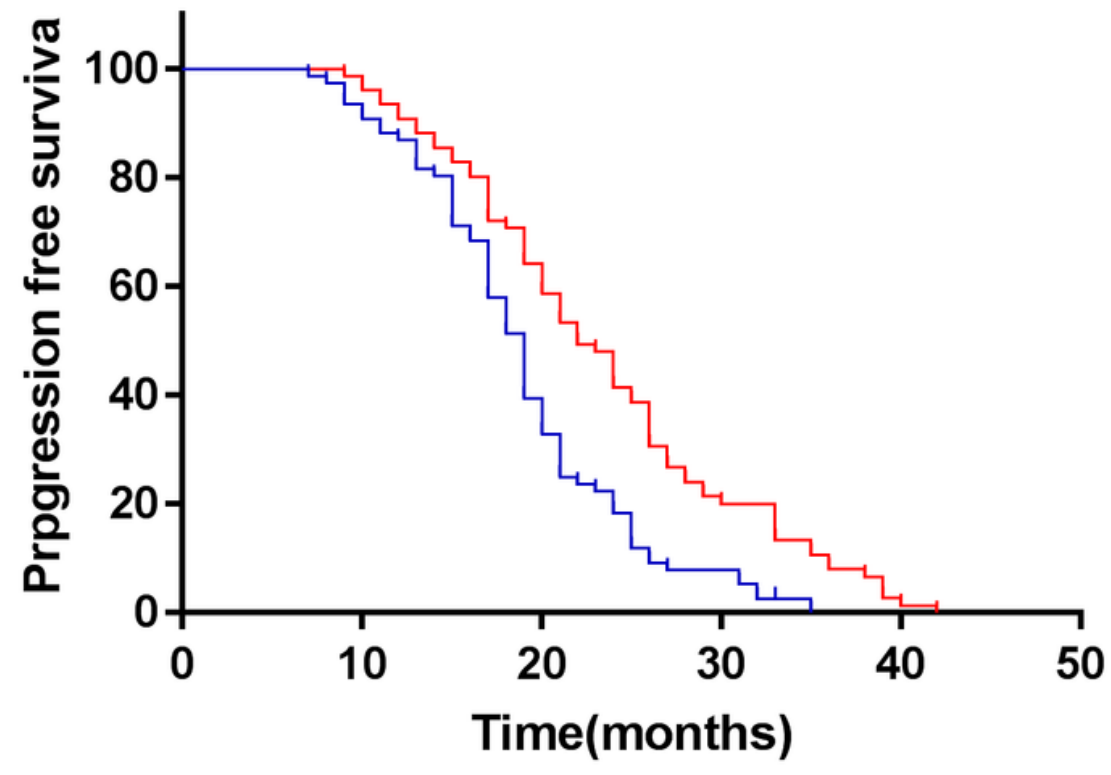

+ IDS alone

$\perp$ IDS+HIPEC

Number at risk

IDS+HIPEC Group

$\begin{array}{llllll}121 & 116 & 75 & 30 & 4 & 0\end{array}$

IDS alone Group

$\begin{array}{llllll}76 & 69 & 25 & 6 & 0 & 0\end{array}$

Figure 1

Progression free survival for the ovarian cancer patients who underwent IDS with or without HIPEC with cisplain. 


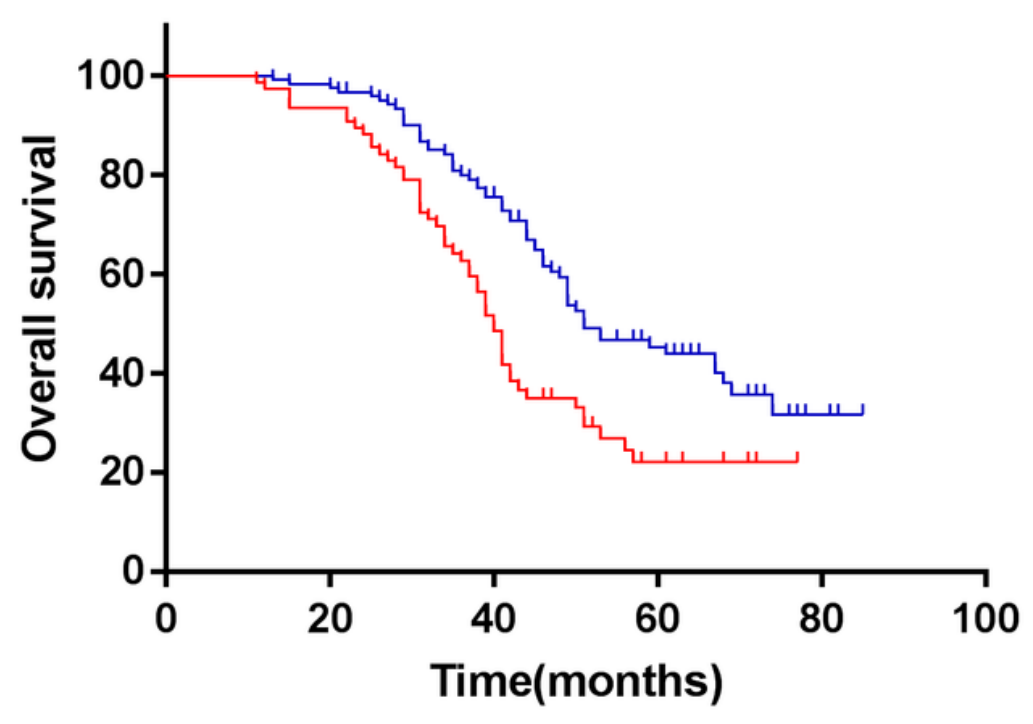

$\perp$ IDS alone

+ IDS+HIPEC

Number at risk

IDS+HIPEC Group

$\begin{array}{llllll}121 & 118 & 79 & 33 & 4 & 0\end{array}$

IDS alone Group

$\begin{array}{llllll}76 & 71 & 29 & 7 & 0 & 0\end{array}$

Figure 2

Overall survival for the patients who underwent IDS with or without HIPEC with cisplatin. 